\title{
Alimentary Tract \\ IgA anti-actin antibodies ELISA in coeliac disease: A multicentre study
}

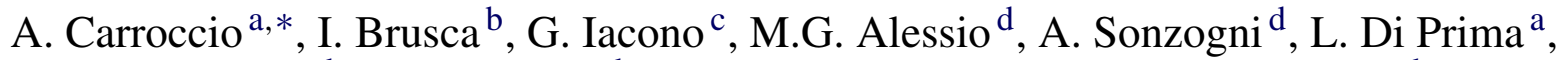

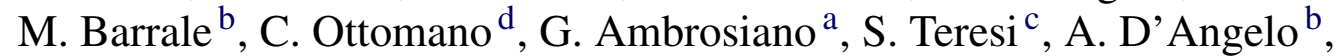

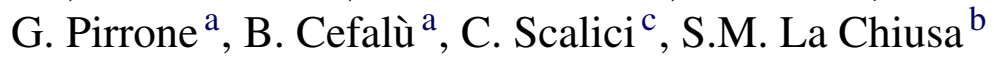 \\ a Internal Medicine, University Hospital, Palermo, via del Vespro 141, 90127 Palermo, Italy \\ b "Buccheri La Ferla" Hospital, Palermo, Italy \\ c Pediatric Gastroenterology, "Di Cristina” Hospital, Palermo, Italy \\ d Bergamo Hospital, Italy \\ Received 20 February 2007; accepted 5 June 2007 \\ Available online 25 July 2007
}

\begin{abstract}
Background. Previous studies have demonstrated that serum anti-actin antibodies are a reliable marker of intestinal damage severity in coeliac disease.

Aims. To validate in a multicentre study the clinical usefulness of serum IgA anti-actin antibody ELISA and its possible use in monitoring intestinal mucosa lesions during gluten-free diet.

Patients and methods. Four centres recruited 205 newly diagnosed coeliac disease patients with villous atrophy, 80 healthy controls and 81 "disease" controls. Twelve coeliac disease patients on gluten-free diet but with persistent symptoms underwent serum IgA anti-actin antibody assay and intestinal histology evaluation. IgA anti-actin antibody ELISA was performed with a commercial kit. All coeliac disease patients underwent intestinal histology study.

Results. IgA anti-actin antibodies showed a sensitivity of $80 \%$ and a specificity of $85 \%$ in the diagnosis of coeliac disease patients with villous atrophy. The area under the receiving operator curve for anti-actin antibodies was 0.873 [95\% C.I. 0.805-0.899]. Serum anti-actin antibodies values were significantly higher in coeliac disease patients than in healthy or "disease" controls $(P<0.0001)$. Serum anti-actin antibodies were positive in 41 of the 60 coeliac disease patients with mild intestinal histology lesions (69\%) and in 123 of the 145 with severe lesions $(85.3 \%)(P<0.05)$. There was a significant inverse correlation between anti-actin antibody values and the villi/crypts ratio $(r=-0.423$; $P<0.0001)$. In the 12 coeliac disease patients on gluten-free diet who underwent re-evaluation as they were persistently symptomatic, intestinal histology showed three cases with persistent villous atrophy: all of these were positive for serum anti-actin antibodies ELISA, whereas both serum anti-tTG and EmAs were negative. The other nine patients showed normal intestinal villi and were negative for serum anti-actin antibodies.
\end{abstract}

Conclusions. Anti-actin antibodies are a reliable marker of severe intestinal mucosa damage in coeliac disease patients and a simple ELISA technique offers an accurate method for their determination. These antibodies seem to be a very reliable marker of persistent intestinal damage in coeliac disease patients.

(C) 2007 Editrice Gastroenterologica Italiana S.r.l. Published by Elsevier Ltd. All rights reserved.

Keywords: Anti-actin antibodies; Coeliac disease; Intestinal histology

* Corresponding author. Tel.: +39091 6552860; fax: +390916552936.

E-mail address: acarroccio@ hotmail.com (A. Carroccio).

\section{Introduction}

Coeliac disease (CD) is an immune-mediated enteropathy triggered by gluten ingestion in genetically predisposed individuals. It is nowadays evident that $\mathrm{CD}$ is one of the 
most common life-long diseases, with a frequency around 1:100-1:200 in the general population [1-3]. The enormous increase in CD diagnoses in recent decades is in large part due to the availability of serum tests with excellent diagnostic accuracy, such the anti-endomysium and antitransglutaminase assays [4-6]. However, these assays do not reflect the severity of the intestinal mucosa damage $[7,8]$. In this respect, the immunofluorescence (IF) assay of $\operatorname{IgA}$ anti-actin antibodies (AAA) has been suggested as a useful method [9,10] and we recently described an ELISA which demonstrated to be an accurate assay for their determination [11].

This multicentre study was designed to evaluate the clinical usefulness of serum IgA AAA ELISA in a greater number of $\mathrm{CD}$ patients and its possible use in monitoring intestinal mucosa lesions during gluten-free diet.

\section{Patients and methods}

CD patients were recruited in four centres (three for adults and one for paediatric CD) between January and December 2004. A total of 205 CD patients were enrolled. IgA AAAs were assayed on the stored sera of 155 adult CD patients (50 M, $105 \mathrm{~F}$, median age 24 years, range 18-76 years) and 50 paediatric $C D$ patients $(22 \mathrm{M}, 28 \mathrm{~F}$, median age 16 months, range 1-14 years). The sera were collected at CD diagnosis, after overnight fasting, and frozen at $-80^{\circ} \mathrm{C}$ for a mean time of 14 months (range 12-24 months) before AAA determination. A previous study had shown that preservation at $-80^{\circ} \mathrm{C}$ did not significantly alter results (inter-assay coefficient of variation was 8.5\%) [11]. The CD clinical presentation was typical (malabsorption syndrome) in 105 patients, atypical (absence of intestinal symptoms) in 85 and silent in 15 (patients identified through screening programs) [12].

In all patients, $\mathrm{CD}$ diagnosis was based on serum EmA and anti-tTG positivity associated with evidence of intestinal villi damage (villous height/crypt depth ratio $<3$ ), and in all cases on a subsequent gluten-free diet clinical symptoms disappeared and EmA and anti-tTGs became negative. As "healthy" controls we enrolled 80 subjects (54 adults evaluated for suspected hypercholesterolaemia: $25 \mathrm{M}$ and $29 \mathrm{~F}$, median age 29 years, range 18-59 years; and 26 children with recurrent pharyngo-tonsilitis, $10 \mathrm{M}$ and $16 \mathrm{~F}$, median age 2 years, range 1-11 years). None of the controls had symptoms or laboratory signs suggesting CD diagnosis and all were negative for EmA and anti-tTG assays. A further 81 adult subjects with autoimmune or gastrointestinal diseases other than CD were enrolled as "disease" controls for IgA AAA assay: type 1 autoimmune hepatitis (4 cases), systemic lupus erythematosus (5 cases), Sjogren's disease (4 cases), primitive biliary cirrhosis (2 case), active Crohn's disease (40 cases), active ulcerative colitis (20 cases), multiple food intolerance (6 cases). All these patients were negative for EmA and anti-tTG assays.
Finally, $12 \mathrm{CD}$ patients ( $4 \mathrm{M}, 8 \mathrm{~F}$, median age 25 years, range 18-49 years) on gluten-free diet for a median time of 20 months (range 14-48 months), but with persistent symptoms, underwent serum IgA anti-tTG and AAA assays and gastroduodenoscopy with multiple duodenal biopsy.

Informed consent was obtained from the adult patients involved in the study and from the parents of the paediatric patients. The study was approved by the Ethics Committee of the University Hospital of Palermo.

\subsection{Serology for $C D$ diagnosis and IgA AAA indirect immunofluorescence assay}

IgA EmAs and anti-tTGs were assayed with commercial kits as previously described [6].

IgA AAA enzyme immunoassay (ELISA) was performed in the laboratory of the "Buccheri La Ferla Hospital" of Palermo, using a commercial kit for anti-actin IgG determination (F-Actin Smooth Muscle, INOVA, San Diego, California, US, ref. 708785) and an anti-serum anti-human IgA conjugate (INOVA, ref. 508549). A 1:101 dilution of serum sample from each patient was prepared and $100 \mu \mathrm{L}$ of the diluted serum was added to the wells and incubated for $30 \mathrm{~min}$ at room temperature. The wells were washed three times with buffer. Hundred microlitres of the IgA conjugate was added to each well and the plates were incubated for 30 min and washed again. Hundred microlitres of TMB chromogen was added to each well and incubated in the dark for $30 \mathrm{~min}$ at room temperature. Stopped reactions were read at $450 \mathrm{~nm}$ and $620 \mathrm{~nm}$. The control wells (no serum) introduced in each plate showed an absorbance of $<0.025$. AAA results were expressed as arbitrary units (AU) in comparison with laboratory standards. The reference curve was obtained by pooling 20 sera from patients highly positive for AAAs (positive controls), evaluated by IF, whereas 20 sera negative for AAAs from healthy controls were mixed and used as negative controls. The threshold normal value was established at 5.8 AU, which was equal to the mean value +3 S.D. obtained in healthy subjects.

The examiners were unaware of the diagnoses and the other laboratory test results. Intra- and inter-assay coefficients of variation (CV) were calculated on 20 samples. The method showed a high reproducibility: intra-assay CV $3.4 \%$ and interassay CV $8.8 \%$.

\subsection{Intestinal histology}

At least three biopsy specimens of the second part of the duodenum were obtained from each CD patient and orientated as previously described [6]. Specimens were embedded in paraffin. Slides were stained with haematoxylin and eosin and graded by conventional histology according to Corazza and Villanacci [13] as normal villi and crypts (Grade A), partial villous atrophy (Grade B), total villous atrophy (Grade C). Furthermore, the ratio between villous height and crypt depth was calculated for each sample. Histology 
was described by an examiner unaware of the laboratory test results.

\section{Statistical analysis}

We followed the STARD checklist for studies on the diagnostic accuracy of tests [14].

The percentage (and 95\% C.I.) of IgA AAA positive results was calculated in the $C D$ patients, controls and in the CD patients with partial or total villous atrophy. The chi-square test was used for frequency analysis. The Mann-Whitney $U$-test was used to compare the serum IgA AAA ELISA results in CD patients and in the control subjects. Spearman's $r$ correlation coefficient was used to evaluate the association of serum AAA values with severity of intestinal mucosa damage (villi/crypts ratio). In addition, receiving operator curves (ROCs) were plotted to show the discriminative ability of the tests. The model plots sensitivity (the proportion of $\mathrm{CD}$ patients attributed by the test to the $\mathrm{CD}$ group) versus 1-specificity (the proportion of control patients attributed by the tests to the $\mathrm{CD}$ group). The areas under the ROC curves and their $95 \%$ confidence intervals were also calculated using the non-parametric method described by Hanley and McNeil [15], developed as a statistical program.

\section{Results}

Fig. 1 shows the individual IgA AAA values in the study groups. Serum IgA AAA evaluated by ELISA were positive in 164 of the 205 (80\%, 95\% C.I. 72.2-87.8) untreated CD patients. Twenty-nine adults and 12 children with CD were negative. Among the 80 healthy controls, 1 subject was positive for serum AAAs, whereas in the group of "disease controls" 23 of the 81 patients were positive. On the basis of the above results, AAAs showed a sensitivity of $80 \%$ (95\% C.I. $73-88.6 \%$ ) and, considering as a single

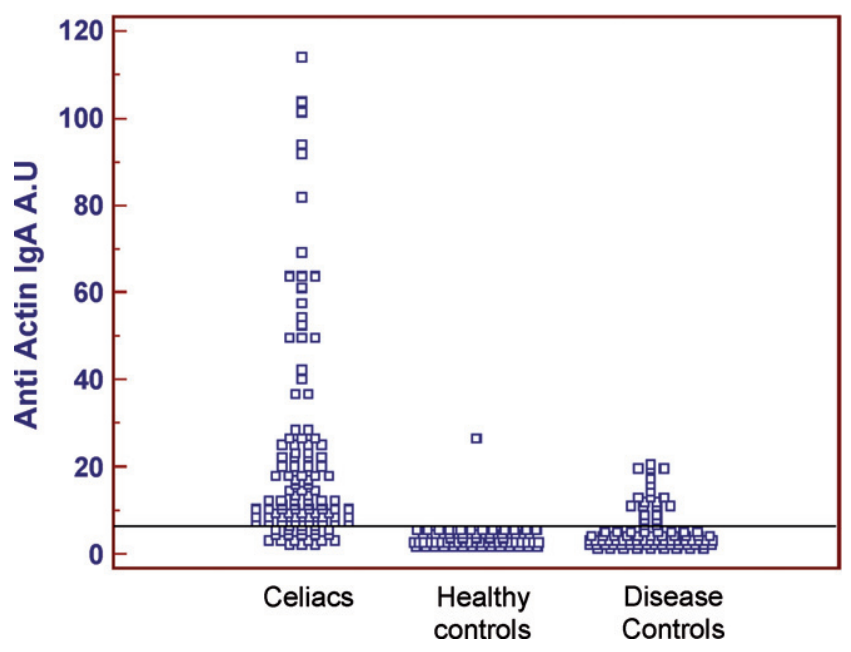

Fig. 1. Individual serum IgA AAA values in the three studied groups.

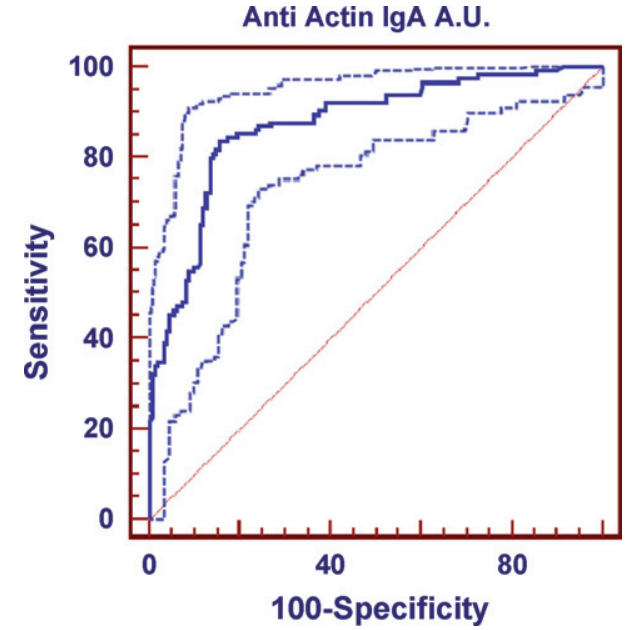

Fig. 2. Receiving operator curve (ROC) for serum IgA anti-actin performed after cumulative analysis of the data from $\mathrm{CD}$ patients and the whole group of control (healthy plus "disease" controls).

group both the healthy and "disease" controls, a specificity of $85 \%$ (95\% C.I. $76-91 \%$ ). The specificity went down to $72 \%$ when exclusively the "disease controls" were considered. After performing a cumulative analysis of the data from the patients and healthy plus "disease" controls, we plotted the ROC curve for AAAs: the area under curve was 0.873 [95\% C.I. 0.805-0.899] (Fig. 2). However, when considering in the cumulative analysis the data from the $\mathrm{CD}$ patients and from the patients with non-CD autoimmune diseases or enteropathy ("disease" controls), the area under the curve was significantly lower: 0.836 (95\% C.I. $0.778-0.885 ; P=0.05)$.

Serum AAAs values were significantly higher in the CD patients (median value 17.7 AU, range 3-111) than in healthy controls (median value 2.4 AU, range 1.1-23.3) $(z=13.34$, $P<0.0001$ ) and "disease" controls (median value 3.8 , range $1.1-21.6 \mathrm{AU})(z=9.32, P<0.0001)$.

Intestinal histology of the $\mathrm{CD}$ patients revealed that 60 had partial villous atrophy (Grade B) and 145 had total villous atrophy (Grade C). Serum AAAs were positive in 41 of the 60 CD patients with Grade B lesions (69\%, 95\% C.I. 59.9-78\%) and in 123 of the 145 with Grade C lesions ( $85.3 \%$, 95\% C.I. 78.4-92.4) $(P<0.01)$. Table 1 resumed the percentage of positive IgA AAA in the study groups. Furthermore, there was a significant inverse correlation between AAA values and the villi/crypts ratio in each patient with $r=-0.423(P<0.0001)$. Anti-tTG values and EmA titer showed a significant but lower inverse correlation with severity of intestinal mucosa damage (for anti-tTGs: $r=-0.381$; for EmAs: $r=-0.328$ ).

Table 2 summarizes the clinical characteristics, intestinal histology findings and serum assay results of the 12 CD patients on gluten-free diet who underwent re-evaluation as they were persistently symptomatic. Intestinal histology showed three cases with persistent villous atrophy (total in one patient and partial in two): all these were positive for serum AAAs ELISA, whereas both serum anti-tTG and EmAs were negative. These three patients declared they 
Table 1

Number of cases and percentages of positive IgA anti-actin antibodies in the different study groups: coeliac patients (according to the grade of intestinal damage), healthy controls and "disease" controls

\begin{tabular}{llll}
\hline CD with partial villi atrophy & CD with total villi atrophy & Healthy controls & "Disease" controls \\
\hline $41 / 60(69 \%)$ & $123 / 145(85.3 \%)$ & $1 / 80(1.3 \%)$ & $23 / 81(28 \%)$ \\
\hline
\end{tabular}

were strictly adhering to the gluten-free diet. The other nine patients showed normal intestinal villi, although most of them showed an elevated number of intra-epithelial lymphocytes. All these nine cases had negative serum AAAs, anti-tTG and EmAs.

\section{Discussion}

The best serological approach to diagnose $\mathrm{CD}$ is based on the EmA and anti-tTG assays, which have a very high diagnostic accuracy [4-6,16,17]. However, these serological tests do not correlate with histopathological features $[8,18]$ and only recently another test - the AAA assay - has been proposed as a marker of the severity of intestinal mucosa damage in CD $[9,10]$.

In the present multicentre study we assayed IgA AAAs with an ELISA which had demonstrated to be an accurate method for AAA determination [11]. Our results showed that in a group of CD patients, all positive for EmA and anti-tTG assays, serum IgA AAAs had $80 \%$ sensitivity and $82 \%$ specificity, thus we confirmed that they are less accurate than the traditional assays in CD diagnosis. Furthermore, it must be underlined that all the CD patients included in this study had various degree of villi atrophy and, as regards the test specificity, it is known that serum AAAs can be detected in patients with autoimmune diseases and in healthy subjects [19]. However, one study reported cases of CD patients negative for serum EmA and positive for IgA AAAs and suggested that this combination, EmA-/AAA+, could indicate complicated CD cases [20]. Others suggested that serum IgA AAA assay associated with other antibodies assays could substitute the EmA assay [21]. Our results, however, are in agreement with those reported by other Italian studies which showed that IgA AAA assay cannot replace EmA and anti-tTG in the diagnostic algorithm of CD [10,22]. Furthermore, we confirmed that serum AAA positivity is indicative of more severe intestinal histology damage. In fact, serum AAAs were found more frequently positive in the CD patients with total villous atrophy $(85 \%)$ than in $C D$ patients with partial villous atrophy (69\%) and there was a significant inverse correlation between IgA AAA values and the villi/crypts ratio $(P<0.0001)$ with a correlation coefficient value higher than those shown by anti-tTG and EmA values.

Another important finding was IgA AAA positivity in the CD patients on gluten-free diet who showed persistent symptoms associated with intestinal mucosa damage. Interestingly, these patients declared they were adhering to the gluten-free diet and both anti-tTG and EmA assays were negative. Although we cannot explain the causes of the persistent histology damage at 14-20 months after commencement of the gluten-free diet, it is relevant that only IgA AAA assay correctly identified these patients and this assay was negative in all the other CD patients with persistent symptoms but contemporary evidence of complete intestinal villi recovery. This seems to confirm that in CD, serum IgA AAAs can be considered a direct expression of severity of intestinal damage and monitoring them could be useful to detect intestinal histology recovery or damage persistence.

IgA AAA ELISA, re-evaluated in the context of a multicentre study, confirmed to be a simple and reliable method. In fact, it was able to detect the antibodies which react against F-actin, the polymerized form of actin which in vitro study

Table 2

Clinical characteristics, intestinal histology findings and serum assay results of the 12 CD patients on gluten-free diet who underwent re-evaluation

\begin{tabular}{|c|c|c|c|c|c|c|}
\hline Patient & GFD duration & Symptoms causing the re-evaluation & Intestinal histology & Serum anti-tTG & Serum EmA & Serum AAA \\
\hline 1 & 20 & Anaemia, diffuse abdominal pain & Grade B & Negative & Absent & Positive \\
\hline 2 & 48 & Dyspepsia & Grade A & Negative & Absent & Negative \\
\hline 3 & 20 & Dyspepsia, anaemia & Grade A & Negative & Absent & Negative \\
\hline 4 & 14 & Weight loss & Grade C & Negative & Absent & Positive \\
\hline 5 & 19 & Anaemia & Grade A & Negative & Absent & Negative \\
\hline 6 & 17 & Dyspepsia & Grade A & Negative & Absent & Negative \\
\hline 7 & 15 & Diarrhoea, anaemia & Grade B & Negative & Absent & Positive \\
\hline 8 & 18 & Diarrhoea, anaemia & Grade A & Negative & Absent & Negative \\
\hline 9 & 30 & Dyspepsia & Grade A & Negative & Absent & Negative \\
\hline 10 & 32 & Dyspepsia & Grade A & Negative & Absent & Negative \\
\hline 11 & 23 & Anaemia & Grade A & Negative & Absent & Negative \\
\hline 12 & 24 & Dyspepsia, anaemia & Grade A & Negative & Absent & Negative \\
\hline
\end{tabular}

Notes: (1) GFD: gluten-free diet; anti-tTG: anti-transglutaminase; EmA: anti-endomysium; AAA: anti-actin. (2) Duration of the GFD diet is given in months. (3) Severity of the intestinal histology damage is given as follows: normal villi and crypts (Grade A), partial villous atrophy (Grade B), total villous atrophy (Grade C) (see reference [13]). 
[23] has shown to be induced in the intestinal epithelial cells of $\mathrm{CD}$ patients minutes after gliadin incubation.

In conclusion, we confirmed that IgA AAAs are a reliable marker of severe intestinal mucosa damage in CD patients and that a simple ELISA technique offers an accurate method for their determination. These antibodies seem to be a very reliable marker of persistent intestinal damage in $\mathrm{CD}$ patients.

\section{Practice points}

- IgA AAAs have been shown in a high percentage of $C D$ patients with severe intestinal histology damage.

- A simple AAA ELISA showed high sensitivity in $C D$ patients, comparable with that reported using the "classical" immunofluorescence technique.

- Using AAA ELISA the frequency of positive assays correlates with the severity of the intestinal damage.

- IgA AAA seems to be a reliable marker of persistent intestinal mucosa damage in CD patients on gluten-free diet.

\section{Research agenda}

- Prospective studies on a greater number of $C D$ patients on gluten-free are needed to better understand the usefulness of AAA assay in monitoring the state of the intestinal mucosa.

- Further studies would be performed to evaluate whether the association of AAA assay with EmA and/or anti-tTG improves the diagnostic accuracy of serologic tests in CD diagnosis.

\section{Conflict of interest statement}

None declared.

\section{Acknowledgements}

We would like to thank Ms. Carole Greenall for her precious revision of the English.

This work was supported by a grant of the Ministry of Universities and Scientific Research (Decr 86 Ric dated 30/01/2002) and of the Ministry of Agriculture of Italy (DM
224/7303/02 dated 10/06/2002) (project "ALICE") and by a grant of the Ministry of Universities and Scientific Research (PRIN 2005, Prof A. Carroccio).

\section{References}

[1] Carlsson AK, Axelsson IE, Borulf SK, Bredberg AC, Ivarsson SA. Serological screening for celiac disease in healthy 2.5 -year-old children in Sweden. Pediatrics 2001;107:42-5.

[2] Not T, Horvath K, Hill ID, Partanen J, Hammed A, Magazzu G, et al. Celiac disease risk in the USA: high prevalence of antiendomysium antibodies in healthy blood donors. Scand J Gastroenterol 1998;33:494-8.

[3] Catassi C, Ratsch IM, Fabiani E, Rossini M, Bordicchia F, Candela F. Celiac disease in the year 2000: exploring the iceberg. Lancet 1994;343:200-3.

[4] Ferreira M, Davies SL, Butler M, Scott D, Clark M, Kumar P. Endomysial antibody: is it the best screening test for coeliac disease? Gut 1992;33:1633-7.

[5] Dieterich W, Laag E, Schopper H, Volta U, Ferguson A, Gillett H, et al. Autoantibodies to tissue transglutaminase as predictors of celiac disease. Gastroenterology 1998;115:1317-21.

[6] Carroccio A, Vitale G, Di Prima L, Chifari N, Napoli S, La Russa C, et al. Comparison of anti-transglutaminase ELISAs and anti-endomysial antibody assay in the diagnosis of celiac disease: a prospective study. Clin Chem 2002;48:1546-50.

[7] Sbarbati A, Valletta E, Bestini M, Cipolli M, Morroni M, Pinelli L, et al. Gluten sensitivity and "normal" histology: is the intestinal mucosa really normal? Dig Liver Dis 2003;35:768-73.

[8] Ciacci C, Cavallaio R, Della Valle N, D'Argenio G. The use of serum tTG-Ab assay in patients on gluten-free diet as a measure of dietetic compliance. Gastroenterology 2002;122:588.

[9] Clemente MG, Musu MP, Frau F, Brusco G, Sole G, Corazza GR, et al. Immune reaction against the cytoskeleton in celiac disease. Gut 2000;47:520-6.

[10] Clemente MG, Musu MP, Troncone R, Volta U, Congia M, Ciacci C, et al. Enterocyte actin antibody detection: a new diagnostic tool in celiac disease diagnosis: results of a multicenter study. Am J Gastroenterol 2004;99:1551-6.

[11] Carroccio A, Brusca I, Iacono G, Di Prima L, Teresi S, Pirrone G, et al. Anti-actin antibodies in celiac disease: correlation with intestinal mucosa damage and comparison of ELISA with the immunofluorescence assay. Clin Chem 2005;51:917-20.

[12] Fasano A, Catassi C. Current approaches to diagnosis and treatment of celiac disease: an evolving spectrum. Gastroenterology 2001;120:636-51.

[13] Corazza GR, Villanacci V. Coeliac disease. Some considerations on the histological diagnosis. Clin Pathol 2005;58:573-4.

[14] Bossuyt PM, Reitsma JB, Bruns DE, Gatsonis CA, Glasziou PP, Irwig LM, et al. Toward complete and accurate reporting of studies of diagnostic accuracy: the STARD initiative. Clin Chem 2003;49:1-6.

[15] Hanley JA, McNeil BJ. A method of comparing the areas under receiver operating characteristic curves derived from the same cases. Radiology 1983;148:839-43.

[16] Hill ID. What are the sensitivity and specificity of serologic tests for celiac disease? Do sensitivity and specificity vary in different populations? Gastroenterology 2005; 128:S25-32.

[17] Rostom A, Dubè C, Cranney A, Saloojee N, Sy R, Garritty C, et al. The diagnostic accuracy of serologic tests for celiac disease: a systematic review. Gastroenterology 2005;128:S38-46.

[18] Dickey W, Hughes DF, McMillan SA. Disappearance of endomysial antibodies in treated celiac disease does not indicate histological recovery. Am J Gastroenterol 2000;95:712-4.

[19] Girard D, Senecal JL. Anti-microfilament IgG antibodies in normal adults and in patients with autoimmune diseases: immunofluorescence 
and immunoblotting analysis of 201 subjects reveals polyreactivity with microfilament-associated proteins. Clin Immunol Immunopathol 1995;74:193-201.

[20] Pedreira S, Sugai E, Moreno ML, Vazquez H, Niveloni S, Smecuol E, et al. Significance of smooth muscle/anti-actin autoantibodies in celiac disease. Acta Gastroenterol Latinoam 2005;35:83-93.

[21] Niveloni S, Kryszak D, Moreno ML, Familiari V, Sapone A, Sugai E, et al. Positive and negative predictive values of a combination of celiac disease serology tests as compared to intestinal histology damage. Gastroenterology 2006;130:A664.

[22] Granito A, Muratori P, Cassani F, Pappas G, Muratori L, Agostinelli D, et al. Anti-actin IgA antibodies in severe coeliac disease. Clin Exp Immunol 2004;137:386-92.

[23] Clemente MG, De Virgilis S, Kang JS, Macatagney R, Musu MP, Di Pierro MR, et al. Early effects of gliadin on enterocyte intracellular signalling involved in intestinal barrier function. Gut 2003;52:218-23. 